\title{
Mathematical modeling and research of heat and moisture transfer processes in porous media
}

\author{
Normakhmad Ravshanov ${ }^{1 *}$, Istam Shadmanov ${ }^{2}$, Kurash Kubyashev ${ }^{3}$, and Sanjar \\ Khikmatullaev ${ }^{3}$ \\ ${ }^{1}$ Research Institute for the Development of Digital Technologies and Artificial Intelligence, Tashkent, \\ Uzbekistan \\ ${ }^{2}$ Bukhara Branch of the V.I.Romanovsky Institute of Mathematics of the Academy of Sciences of the \\ Republic of Uzbekistan, Bukhara, Uzbekistan \\ ${ }^{3}$ Tashkent Institute of Irrigation and Agricultural Mechanization Engineers, Tashkent, Uzbekistan
}

\begin{abstract}
A mathematical model, numerical algorithm, solution, and results of the computational experiment on a computer are developed to predict the process of heat and moisture transfer in porous media, taking into account such factors as the internal heat and moisture release of a porous natural product on the example of raw cotton and its products of hulling, seeds of various crops. It also considers the effect of temperature and moisture content changes in the environment on the storage and drying of porous materials.

In this study, the developed mathematical support of the object under research makes it possible to predict the change in temperature and moisture content at arbitrary points of the porous body and serves to prevent the loss of quality and spontaneous combustion of materials under solar radiation and to analyze and make managerial decisions.

Based on the method of coordinate-wise splitting, a numerical algorithm for calculating three-dimensional heat and moisture transfer problems in areas of the parallelepiped type is presented. An implicit second-order difference scheme for calculating the required functions is presented.

Based on the numerical calculations performed, it was established that moisture and heat transfer and their exchange with the environment occurs in the upper layers of the cotton pile; in the inner layers, there is an increase in temperature and moisture due to the respiration of raw cotton and its seeds, which depends on the degree of moisture of the raw cotton and their products of hulling.
\end{abstract}

\section{Introduction}

The theory of energy and moisture transfer in a capillary-porous body is of great importance in the technological processes of the food, chemical, construction, and light industries. The modern theory of drying is based on moisture and heat transfer laws in the stored and dried material. Moisture and heat transfer occurs at the growth or downsizes of

*Corresponding author: ravshanzade-09@mail.ru 
humidity and temperature in a porous body. The most efficient moisture and heat transfer in capillary-porous agricultural products are due to the correct combination of the material's heat temperatures and moisture content. This condition is determinant for maintaining the viability of seeds and the quality of agricultural products.

A great contribution to the formation and development of the theory of heat and mass transfer was made by A.N. Tikhonov, A.A. Samarsky, A.V. Lykov, Yu.A. Mikhailov, B.M.Budak, D. Anderson, J. Tannekhil, AI Leontiev, BN Yudaev, A.Yu. Krainov, and others. The scientists A.V. Lykov, V.M. Paskanov, V.P. Isachenko, V.A. Osipova, A.S. Sukomel, P. S. Kossovich, A. V. Lebedev, V. G. Goryachkin, M. V. Kirpichev, and others developed the basic provisions of the drying process of colloidal capillary-porous bodies.

At present, several models are used to describe the processes of heat and moisture transfer in capillary-porous media; the most studied are the models proposed by Philip and De Vries [1], Lykov [2], and Whitaker [3]. These models were developed on the basis and principles of conservation of heat and mass, Fourier's law for thermal conductivity, Fick's and Darcy's laws for gas and fluid diffusion, respectively. A feature of these models is the choice of control potentials such as partial pressure, relative humidity, and water content in porous bodies.

A.V. Lykov and Yu.A. Mikhailov [4] gave an extended system of potential conductivity with the equation for static pressure, based on the surface tension forces of fluid and the laws of capillarity.

Lykov A.V. has stated that under intense heating of a capillary porous body, the drying kinetics can depend not only on the gradient of the moisture transfer potential but also on the temperature gradient and the internal pressure gradient. The system of differential equations describing non-stationary fields of moisture content, temperature, and pressure inside a wet body during the drying process has the following form [5]:

$$
\begin{aligned}
& \frac{\partial u}{\partial \tau}=a_{11} \nabla^{2} u+a_{12} \nabla^{2} T+a_{13} \nabla^{2} P \\
& \frac{\partial T}{\partial \tau}=a_{21} \nabla^{2} u+a_{22} \nabla^{2} T+a_{23} \nabla^{2} P \\
& \frac{\partial P}{\partial \tau}=a_{31} \nabla^{2} u+a_{32} \nabla^{2} T+a_{33} \nabla^{2} P
\end{aligned}
$$

where $a_{11}=\frac{k_{p}}{c_{p} \rho_{0}} ; \quad a_{12}=a_{11} \delta ; a_{13}=\frac{k_{p}}{\rho_{0}} ; \quad a_{21}=\frac{r^{*} \varepsilon}{c} a_{11} ; a_{22}=a+\frac{r^{*} \varepsilon}{c} a_{11} \delta ;$
$a_{23}=\frac{r^{*} \varepsilon}{c} a_{11} \delta_{p} ; \quad a_{31}=\frac{\varepsilon a_{11}}{c_{p}} ; \quad a_{32}=-\frac{r^{*} a_{11}}{c_{p}} \delta ; \quad a_{33}=D_{p}-\frac{\varepsilon a_{11}}{c_{p}} \delta_{p} ; \quad c_{p}-$ is the coefficient of the capacity of moist air in a porous body; $k_{p}-$ is the coefficient of filtration moisture transfer; $D_{p}$-is the coefficient of convective filtration diffusion; $\rho_{0}-$ is the density of the dry skeleton of the body; $\nabla^{2}-$ is the Laplace operator.

The biggest problem when using Lykov's equations is to determine coefficients $a_{i, j}$. In practice, it is often impossible to obtain these coefficients for solving the complete system 
of equations. Irudayaraj and $\mathrm{Wu}[6]$ and Lewis and Ferguson [7] were the first who have numerically investigated the solution of Lykov's partial differential equations. These equations are widely used and solved by the finite element method.

In particular, the study in [8] gives a numerical scheme for the coupled solution of the Lykov and Maxwell equations of heat and moisture propagation. It is built based on two algorithms: the problem of calculating the density field of electromagnetic losses, reflection and transmission coefficients is solved for a given distribution of the dielectric constant; the problem of calculating the fields of temperature and moisture content is solved for a given density field of electromagnetic losses. A numerical experiment was conducted, the results of which were in good agreement with the experimental data available in the literature.

An eigenvalue analysis approach is used in [9] for solving a system of Lykov linear partial differential equations aimed at the most general type of boundary conditions. Solutions for the dimensionless distribution of temperature and moisture, obtained using the matrix calculus, form general expressions, from which, as a special case, solutions can be obtained for many specific one-dimensional, time-dependent problems of heat and mass transfer. A specific example of contact drying of a wet porous sheet with a uniform distribution of initial temperature and moisture is considered.

In [10], a generalized mathematical model of heat and moisture processes during thermal treatment of wood is proposed. The mathematical model is based on a set of coupled heat and mass transfer equations proposed by Lykov. Average moisture and temperature profiles were predicted as a function of time and governing parameters. It is shown that the governing dimensionless parameters have a substantial effect on the kinetics of heat and moisture transfer. The results obtained within the framework of the considered model demonstrate realistic physical behavior.

A numerical study of three-dimensional heat and moisture transfer in capillary-porous media using a variable number of phase transformations is proposed in [11]. The influence of the number of phase transformations as a variable parameter on the temperature and moisture potential was sought for various values of the main dimensionless parameters of the Lykov system of equations. It was found that for lower values of these dimensionless parameters, the assumption of a constant number of phase transformations is logical, but for higher values of $L u, K o$, and $P n$, more exact modeling is required, and a variable number of phase transformations can be used to obtain better agreement with experimental data.

Mathematical modeling of heat and mass transfer processes during drying and storage of various products, such as raw cotton, fruit, wood, and others, was conducted in [12][20].

According to the above, Lykov's theory is a well-established model for considering simultaneous heat and mass transfer during the drying process. That's why Lykov's model is taken as a basic model for the numerical simulation of the process of heat and moisture transfer in porous media.

\subsection{Statement of problem}

Taking into account the main indices of the process of drying and storage of porous bodies, a mathematical model of heat and moisture transfer, considering the sources of heat and moisture (in contrast to the above studies) is described using systems of partial differential equations:

$$
\frac{\partial T}{\partial \tau}=a_{11}\left(\frac{\partial^{2} T}{\partial x^{2}}+\frac{\partial^{2} T}{\partial y^{2}}+\frac{\partial^{2} T}{\partial z^{2}}\right)+a_{12}\left(\frac{\partial^{2} u}{\partial x^{2}}+\frac{\partial^{2} u}{\partial y^{2}}+\frac{\partial^{2} u}{\partial z^{2}}\right)+f
$$




$$
\frac{\partial u}{\partial \tau}=a_{21}\left(\frac{\partial^{2} u}{\partial x^{2}}+\frac{\partial^{2} u}{\partial y^{2}}+\frac{\partial^{2} u}{\partial z^{2}}\right)+a_{22}\left(\frac{\partial^{2} T}{\partial x^{2}}+\frac{\partial^{2} T}{\partial y^{2}}+\frac{\partial^{2} T}{\partial z^{2}}\right)+q
$$

with initial and boundary conditions, respectively:

$$
\begin{aligned}
& T(x, y, z, 0)=T_{0}(x, y, z), u(x, y, z, 0)=u_{0}(x, y, z) \text {; } \\
& \left.\lambda_{1} \frac{\partial T}{\partial x}\right|_{x=0}=-\beta_{1}\left(T_{o c}-T\right)-\eta \rho \gamma R(\tau) \\
& \left.\lambda_{1} \frac{\partial T}{\partial x}\right|_{x=L_{x}}=-\beta_{1}\left(T_{o c}-T\right)-\eta \rho \gamma R(\tau) \text {; } \\
& \left.\lambda_{1} \frac{\partial T}{\partial y}\right|_{y=0}=-\beta_{1}\left(T_{o c}-T\right)-\eta \rho \gamma R(\tau) \\
& \left.\lambda_{1} \frac{\partial T}{\partial y}\right|_{y=L y}=-\beta_{1}\left(T_{o c}-T\right)-\eta \rho \gamma R(\tau) ; \\
& \left.\frac{\partial T}{\partial z}\right|_{z=0}=0 ; \\
& \left.\lambda_{1} \frac{\partial T}{\partial z}\right|_{z=L_{z}}=-\beta_{1}\left(T_{o c}-T\right)-\eta \rho \gamma R(\tau) \\
& \left.\lambda_{2} \frac{\partial u}{\partial x}\right|_{x=0}=-\beta_{2}\left(u_{o c}-u(0, y, z, \tau)\right) \\
& \left.\lambda_{2} \frac{\partial u}{\partial x}\right|_{x=L_{x}}=-\beta_{2}\left(u_{o c}-u\left(L_{x}, y, z, \tau\right)\right) \\
& \left.\lambda_{2} \frac{\partial u}{\partial y}\right|_{y=0}=-\beta_{2}\left(u_{o c}-u(x, 0, z, \tau)\right) \\
& \left.\lambda_{2} \frac{\partial u}{\partial y}\right|_{y=L_{y}}=-\beta_{2}\left(u_{o c}-u\left(x, L_{y}, z, \tau\right)\right) \\
& \left.\frac{\partial u}{\partial z}\right|_{z=0}=0 \\
& \left.\lambda_{2} \frac{\partial u}{\partial z}\right|_{z=L_{z}}=-\beta_{2}\left(u_{o c}-u\left(x, y, L_{z}, \tau\right)\right) \text {. }
\end{aligned}
$$


Here $T$ and $u$ are the changes in temperature and moisture content of a porous body; $a_{11}=\frac{\lambda_{1}}{\rho c_{1}} ; a_{12}=\frac{\lambda_{2}}{\rho c_{2}}\left(\mathrm{~m}^{2} / \mathrm{s}\right)$ are the coefficients of thermal diffusivity and moisture conductivity; $\lambda_{1}, \lambda_{2}$ are the coefficients of thermal conductivity and moisture conductivity; $\rho$ is the body density $\left(\mathrm{kg} / \mathrm{m}^{3}\right)$; specific heat $c_{1}$ and moisture capacity $c_{2}(J /(k g \cdot K)) ; f(x, y, z, \tau)=b \cdot e^{-\alpha \tau}$ is the intensity of internal heat release of the mass $\left(K s^{-1}\right) ; b=\frac{u}{c_{1}}$ is the heat release coefficient, which depends on the moisture content of porous bodies, so, $b=f(u(x, y, z, t)) ; \alpha$ is the empirical parameter; $q(x, y, z, \tau)=\rho m_{0} e^{-\xi \tau}$ the intensity of internal moisture sources; at constant values of the material density $\rho\left(\mathrm{kg} / \mathrm{m}^{3}\right) ; \xi$ is the drying coefficient $(1 / \mathrm{sec}) ; m_{0}$ is the maximum evaporation rate $\left(\mathrm{kg} / \mathrm{m}^{2} \cdot \mathrm{sec}\right)$. It should be noted that the evaporation rate is calculated using $m=m_{0} e^{\xi \tau}$. If to assume that $\xi=0$ then $m=m_{0}=$ const, we get a constant evaporation rate corresponding to the first drying period; $\beta_{1}$ is the heat transfer coefficient between the mass and the air surrounding it; $T_{o c}$ is the ambient temperature; $\eta$ are the coefficients to reduce the boundary condition to dimensional form; $\gamma$ is the coefficient of sunlight absorption by the material; $R(\tau)$ is the insolation of the flow of solar radiation on the surface of the stored material; $\beta_{2}$ is the moisture transfer coefficient between the mass and the air surrounding it; $u_{o c}$ is the moisture content of the ambient.

The external shape of the porous body is taken as a rectangular parallelepiped. The parallelepiped is located in the first octant of the Cartesian coordinate system, and its dimensions in terms of coordinates are $L_{x}, L_{y}, L_{z}$.

\section{Methods}

Since the formulated problem (1) - (15) is described by a multidimensional partial differential equation with the corresponding initial and boundary conditions, it is practically impossible to obtain its exact solution in analytical form. Considering the above circumstances, we use the method of splitting by coordinates at each time layer to solve the problem.

The method of splitting into physical processes is based on a high-order approximation [21], substantiating the additivity of processes for sufficiently short time steps [22] and proving the original equation by a total approximation due to splitting. The general theory of splitting is completely described in [23], [24], and the features of splitting for the convection problems in rectangular regions and parallelepipeds are presented in [25], [26].

Within the framework of the theory of the splitting by coordinates, the method for solving the problem (1) - (15) can be represented in the form of three consecutive subproblems. 
Problem A:

$$
\begin{gathered}
\frac{\partial T}{\partial \tau}=a_{11} \frac{\partial^{2} T}{\partial x^{2}}+a_{12} \frac{\partial^{2} u}{\partial x^{2}}+\frac{1}{3} f \\
\frac{\partial u}{\partial \tau}=a_{21} \frac{\partial^{2} u}{\partial x^{2}}+a_{22} \frac{\partial^{2} T}{\partial x^{2}}+\frac{1}{3} q
\end{gathered}
$$

With initial conditions:

$$
T_{1}^{0}=T_{3}^{n+1}, u_{1}^{0}=u_{3}^{n+1} \text { at } \tau=\tau_{n}
$$

and boundary conditions

$$
\begin{aligned}
& \left.\lambda_{1} \frac{\partial T}{\partial x}\right|_{x=0}=-\beta_{1}\left(T_{o c}-T_{1}\right)-\eta \rho \gamma R(\tau) \\
& \left.\lambda_{1} \frac{\partial T}{\partial x}\right|_{x=L_{x}}=-\beta_{1}\left(T_{o c}-T_{1}\right)-\eta \rho \gamma R(\tau) \\
& \left.\lambda_{2} \frac{\partial u}{\partial x}\right|_{x=0}=-\beta_{2}\left(u_{o c}-u_{1}\right) \\
& \left.\lambda_{2} \frac{\partial u}{\partial x}\right|_{x=L_{x}}=-\beta_{2}\left(u_{o c}-u_{1}\right)
\end{aligned}
$$

Problem B:

$$
\begin{aligned}
& \frac{\partial T}{\partial \tau}=a_{11} \frac{\partial^{2} T}{\partial y^{2}}+a_{12} \frac{\partial^{2} u}{\partial y^{2}}+\frac{1}{3} f \\
& \frac{\partial u}{\partial \tau}=a_{21} \frac{\partial^{2} u}{\partial y^{2}}+a_{22} \frac{\partial^{2} T}{\partial y^{2}}+\frac{1}{3} q
\end{aligned}
$$

With initial conditions:

$$
T_{2}^{0}=T_{1}^{n+1}, u_{2}^{0}=u_{1}^{n+1}
$$

and boundary conditions

$$
\begin{aligned}
& \left.\lambda_{1} \frac{\partial T}{\partial y}\right|_{y=0}=-\beta_{1}\left(T_{o c}-T_{2}\right)-\eta \rho \gamma R(\tau) \\
& \left.\lambda_{1} \frac{\partial T}{\partial y}\right|_{y=L_{y}}=-\beta_{1}\left(T_{o c}-T_{2}\right)-\eta \rho \gamma R(\tau) \\
& \left.\lambda_{2} \frac{\partial u}{\partial y}\right|_{y=0}=-\beta_{2}\left(u_{o c}-u_{2}\right)
\end{aligned}
$$




$$
\left.\lambda_{2} \frac{\partial u}{\partial y}\right|_{y=L_{y}}=-\beta_{2}\left(u_{o c}-u_{2}\right)
$$

Problem C:

$$
\begin{aligned}
& \frac{\partial T}{\partial \tau}=a_{11} \frac{\partial^{2} T}{\partial z^{2}}+a_{12} \frac{\partial^{2} u}{\partial z^{2}}+\frac{1}{3} f \\
& \frac{\partial u}{\partial \tau}=a_{21} \frac{\partial^{2} u}{\partial z^{2}}+a_{22} \frac{\partial^{2} T}{\partial z^{2}}+\frac{1}{3} q
\end{aligned}
$$

With initial conditions:

$$
T_{3}^{0}=T_{2}^{n+1}, u_{3}^{0}=u_{2}^{n+1}
$$

And boundary conditions:

$$
\begin{aligned}
& \left.\lambda_{1} \frac{\partial T}{\partial z}\right|_{z=0}=0 \\
& \left.\lambda_{1} \frac{\partial T}{\partial z}\right|_{z=L_{z}}=-\beta_{1}\left(T_{o c}-T_{3}\right)-\eta \rho \gamma R(\tau) ; \\
& \left.\lambda_{2} \frac{\partial u}{\partial z}\right|_{z=0}=0 \\
& \left.\lambda_{2} \frac{\partial u}{\partial z}\right|_{z=L_{z}}=-\beta_{2}\left(u_{o c}-u_{3}\right) .
\end{aligned}
$$

The above option of the scheme performs a sequential splitting by coordinates of the problem, starting with the direction $\overrightarrow{O x}$, then $\overrightarrow{O y}$, and finally $\overrightarrow{O z}$, within each time interval $\tau_{n} \leq \tau \leq \tau_{n+1}$.

Thus, after splitting the original problem by coordinates, we obtained three subproblems (16) - (22), (23) - (29), and (30) - (36), which we will solve using an implicit finite-difference scheme in time and with the second-order accuracy in coordinates, replacing the continuous region of variation of the sought variables by a grid with steps $\Delta x ; \Delta y ; \Delta z$ :

$$
\begin{aligned}
& \Omega_{x y z t}=\left\{\left(x_{i}=i \Delta x, y_{j}=j \Delta y, z_{k}=k \Delta z, \tau_{n}=n \Delta t\right) ;\right. \\
& \left.i=\overline{1, N_{x}} ; j=\overline{1, M_{y}}, k=\overline{1, L_{z}}, n=\overline{0, N_{t}}, \Delta t=\frac{1}{N_{t}}\right\} .
\end{aligned}
$$


Then, for problem A, equation (16) is approximated in $x$ :

$$
\begin{aligned}
& \frac{1}{2} \frac{T_{1, i}^{n+\frac{1}{3}}-T_{1, i}^{n}}{\Delta \tau / 3}+\frac{1}{2} \frac{T_{1, i+1}^{n+\frac{1}{3}}-T_{1, i+1}^{n}}{\Delta \tau / 3}=a_{11} \frac{T_{1, i+1}^{n+\frac{1}{3}}-2 T_{1, i}^{n+\frac{1}{3}}+T_{1, i-1}^{n+\frac{1}{3}}}{\Delta x^{2}}+ \\
& \quad+a_{12} \frac{u_{1, i+1}^{n}-2 u_{1, i}^{n}+u_{1, i-1}^{n}}{\Delta x^{2}}+\frac{1}{3} f_{i}^{n+\frac{1}{3}}
\end{aligned}
$$

and grouping similar terms in equation (37) we get:

$$
\begin{gathered}
\frac{a_{11}}{\Delta x^{2}} T_{1, i-1}^{n+\frac{1}{3}}-\left(\frac{3}{2 \Delta \tau}+\frac{\left.2 a_{11}\right)}{\Delta x^{2}}\right) T_{1, i}^{n+\frac{1}{3}}+\left(-\frac{3}{2 \Delta \tau}+\frac{a_{11}}{\Delta x^{2}}\right) T_{1, i+1}^{n+\frac{1}{3}}=-\frac{3}{2 \Delta \tau} T_{1, i}^{n}- \\
-\frac{3}{2 \Delta \tau} T_{1, i+1}^{n}-a_{12} \frac{u_{1, i+1}^{n}-2 u_{1, i}^{n}+u_{1, i-1}^{n}}{\Delta x^{2}}-\frac{1}{3} f_{i}^{n+\frac{1}{3}} .
\end{gathered}
$$

Let us introduce the notation:

$$
\begin{aligned}
& a_{1, i}=\frac{a_{11}}{\Delta x^{2}}, \quad b_{1, i}=\frac{3}{2 \Delta \tau}+\frac{2 a_{11}}{\Delta x^{2}}, \quad c_{1, i}=-\frac{3}{2 \Delta \tau}+\frac{a_{11}}{\Delta x^{2}}, \\
& d_{1, i}=\frac{3}{2 \Delta \tau} T_{1, i}^{n}+\frac{3}{2 \Delta \tau} T_{1, i+1}^{n}+a_{12} \frac{u_{1, i+1}^{n}-2 u_{1, i}^{n}+u_{1, i-1}^{n}}{\Delta x^{2}}+\frac{1}{3} f_{i}^{n+\frac{1}{3}}
\end{aligned}
$$

we get a system of three diagonal algebraic equations:

$$
a_{1, i} T_{1, i-1}^{n+\frac{1}{3}}-b_{1, i} T_{1, i}^{n+\frac{1}{3}}+c_{1, i} T_{1, i+1}^{n+\frac{1}{3}}=-d_{1, i}
$$

Now, boundary condition (19) is approximated in $x$ and at $i=1$ we obtain:

$$
\lambda_{1} \frac{-3 T_{1,0}^{n+\frac{1}{3}}+4 T_{1,1}^{n+\frac{1}{3}}-T_{1,2}^{n+\frac{1}{3}}}{2 \Delta x}=-\beta_{1} T_{o c}+\beta_{1} T_{1,0}^{n+\frac{1}{3}}-\varphi^{n}
$$

where $\varphi^{n}=\eta \rho \gamma R(\tau)$.

From the system of equations (38) at $i=1$, we obtain:

$$
a_{1,1} T_{1,0}^{n+\frac{1}{3}}-b_{1,1} T_{1,1}^{n+\frac{1}{3}}+c_{1,1} T_{1,2}^{n+\frac{1}{3}}=-d_{1,1} .
$$

Substituting $T_{1,2}^{n+\frac{1}{3}}$ from (40) into (39), we determine $T_{1,0}^{n+\frac{1}{3}}$ : 


$$
T_{1,0}^{n+\frac{1}{3}}=\frac{\lambda_{1} b_{1,1}-4 \lambda_{1} c_{1,1}}{a_{1,1} \lambda_{1}-3 c_{1,1} \lambda_{1}-2 \Delta x c_{1,1} \beta_{1}} T_{1,1}^{n+\frac{1}{3}}+\frac{-d_{1,1} \lambda_{1}-2 \Delta x c_{1,1} \beta_{1} T_{o c}-2 \Delta x c_{1,1} \varphi^{n}}{a_{1,1} \lambda_{1}-3 c_{1,1} \lambda_{1}-2 \Delta x c_{1,1} \beta_{1}}
$$

Introducing the notation with (41) we obtain:

$$
T_{1,0}^{n+\frac{1}{3}}=\alpha_{1,0} T_{1,1}^{n+\frac{1}{3}}+\beta_{1,0}
$$

From relation (42), it follows that the sweep coefficients $\alpha_{1,0}$ and $\beta_{1,0}$ are calculated as:

$$
\alpha_{1,0}=\frac{\lambda_{1} b_{1,1}-4 \lambda_{1} c_{1,1}}{a_{1,1} \lambda_{1}-3 c_{1,1} \lambda_{1}-2 \Delta x c_{1,1} \beta_{1}} \text { and } \beta_{1,0}=\frac{-d_{1,1} \lambda_{1}-2 \Delta x c_{1,1} \beta_{1} T_{o c}-2 \Delta x c_{1,1} \varphi^{n}}{a_{1,1} \lambda_{1}-3 c_{1,1} \lambda_{1}-2 \Delta x c_{1,1} \beta_{1}} .
$$

Similarly, approximating the boundary condition (20) in $x$ and for $i=N$, we obtain:

$$
\lambda_{1} \frac{T_{1, \mathrm{~N}-2}^{n+\frac{1}{3}}-4 T_{1, \mathrm{~N}-1}^{n+\frac{1}{3}}+3 T_{1, \mathrm{~N}}^{n+\frac{1}{3}}}{2 \Delta x}=-\beta_{1} T_{o c}+\beta_{1} T_{1, N}^{n+\frac{1}{3}}-\varphi^{n}
$$

where $\varphi^{n}=\eta \rho \gamma R(\tau)$

Using the reverse sweep method for the sequence $N, N-1$, and $N-2$, we determine $T_{1, \mathrm{~N}-1}^{n+\frac{1}{3}}$ and $T_{1, \mathrm{~N}-2}^{n+\frac{1}{3}}$ :

$$
\begin{gathered}
T_{1, \mathrm{~N}-1}^{n+\frac{1}{3}}=\alpha_{1, \mathrm{~N}-1} T_{1, N}^{n+\frac{1}{3}}+\beta_{1, \mathrm{~N}-1}, \\
T_{1, \mathrm{~N}-2}^{n+\frac{1}{3}}=\alpha_{1, \mathrm{~N}-2} \alpha_{1, \mathrm{~N}-1} T_{1, N}^{n+\frac{1}{3}}+\alpha_{1, \mathrm{~N}-2} \beta_{1, \mathrm{~N}-1}+\beta_{1, \mathrm{~N}-2} .
\end{gathered}
$$

Substituting $T_{1, \mathrm{~N}-1}^{n+\frac{1}{3}}$ from (44) and $T_{1, \mathrm{~N}-2}^{n+\frac{1}{3}}$ from (45) into (43) we determine $T_{1, \mathrm{~N}}^{n+\frac{1}{3}}$ :

$$
T_{1, \mathrm{~N}}^{n+\frac{1}{3}}=\frac{-\lambda_{1} \alpha_{1, \mathrm{~N}-2} \beta_{1, \mathrm{~N}-1}-\lambda_{1} \beta_{1, \mathrm{~N}-2}+4 \lambda_{1} \beta_{1, \mathrm{~N}-1}-2 \Delta x \beta_{1} T_{o c}-2 \Delta x \varphi^{n}}{3 \lambda_{1}-2 \Delta x \beta_{1}+\lambda_{1} \alpha_{1, \mathrm{~N}-2} \alpha_{1, \mathrm{~N}-1}-4 \lambda_{1} \alpha_{1, \mathrm{~N}-1}}
$$

The values of the temperature sequence $T_{1, \mathrm{~N}-1}^{n+\frac{1}{3}}, T_{1, \mathrm{~N}-2}^{n+\frac{1}{3}}, \ldots, T_{1,1}^{n+\frac{1}{3}}$ are determined by the reverse sweep method by decreasing $i$ sequence:

$$
T_{1, i}^{n+\frac{1}{3}}=\alpha_{1, i} T_{1, i+1}^{n+\frac{1}{3}}+\beta_{1, i}, \quad i=\overline{N-1,1} .
$$


Next, equation (17) is approximated in $x$ :

$$
\begin{aligned}
& \frac{1}{2} \frac{u_{1, i}^{n+\frac{1}{3}}-u_{1, i}^{n}}{\Delta \tau / 3}+\frac{1}{2} \frac{u_{1, i+1}^{n+\frac{1}{3}}-u_{1, i+1}^{n}}{\Delta \tau / 3}=a_{21} \frac{\begin{array}{c}
n+\frac{1}{3} \\
u_{1, i+1}-2 u_{1, i}{ }^{n+\frac{1}{3}}+u_{1, i-1}
\end{array}}{\Delta x^{2}}+ \\
& +a_{22} \frac{T_{1, i+1}^{n}-2 T_{1, i}^{n}+T_{1, i-1}^{n}}{\Delta x^{2}}+\frac{1}{3} q_{i}^{n+\frac{1}{3}} \text {; }
\end{aligned}
$$

and grouping similar terms into equation (48) we get:

$$
\begin{aligned}
\frac{a_{21}}{\Delta x^{2}} u_{1, i-1}^{n+\frac{1}{3}}- & \left(\frac{3}{2 \Delta \tau}+\frac{2 a_{21}}{\Delta x^{2}}\right) u_{1, i}^{n+\frac{1}{3}}+\left(-\frac{3}{2 \Delta \tau}+\frac{a_{21}}{\Delta x^{2}}\right) u_{1, i+1}^{n+\frac{1}{3}}=-\frac{3}{2 \Delta \tau} u_{1, i}^{n}- \\
& -\frac{3}{2 \Delta \tau} u_{1, i+1}^{n}-a_{22} \frac{T_{1, i+1}^{n}-2 T_{1, i}^{n}+T_{1, i-1}^{n}}{\Delta x^{2}}-\frac{1}{3} q_{i}^{n+\frac{1}{3}}
\end{aligned}
$$

Let us introduce the notation:

$$
\begin{gathered}
\hat{a}_{1, i}=\frac{a_{21}}{\Delta x^{2}}, \quad \hat{b} 1, i=\frac{3}{2 \Delta \tau}+\frac{2 a_{21}}{\Delta x^{2}}, \quad c_{1, i}=-\frac{3}{2 \Delta \tau}+\frac{a_{21}}{\Delta x^{2}} \\
\hat{d}_{1, i}=\frac{3}{2 \Delta \tau} u_{1, i}^{n}+\frac{3}{2 \Delta \tau} u_{1, i+1}^{n}+a_{22} \frac{T_{1, i+1}^{n}-2 T_{1, i}^{n}+T_{1, i-1}^{n}}{\Delta x^{2}}+\frac{1}{3} q_{i}^{n+\frac{1}{3}}
\end{gathered}
$$

we get a system of three diagonal algebraic equations:

$$
\widehat{a}_{1, i} u_{1, i-1}^{n+\frac{1}{3}}-\widehat{b}_{1, i} u_{1, i}^{n+\frac{1}{3}}+\widehat{c}_{1, i} u_{1, i+1}^{n+\frac{1}{3}}=-d_{1, i}
$$

Now, boundary condition (21) is approximated in $x$ and at $i=1$ we obtain:

$$
\lambda_{2} \frac{-3 u_{1,0}^{n+\frac{1}{3}}+4 u_{1,1}^{n+\frac{1}{3}}-u_{1,2}^{n+\frac{1}{3}}}{2 \Delta x}=-\beta_{2} u_{o c}+\beta_{2} u_{1,0}^{n+\frac{1}{3}}
$$

from the system of equations (38) at $i=1$, we obtain:

$$
\hat{a}_{1,1} u_{1,0}^{n+\frac{1}{3}}-\widehat{b}_{1,1} u_{1,1}^{n+\frac{1}{3}}+\hat{c}_{1,1} u_{1,2}^{n+\frac{1}{3}}=-d_{1,1}
$$

substituting $u_{1,2}^{n+\frac{1}{3}}$ from (51) into (50) we determine $u_{1,0}^{n+\frac{1}{3}}$ : 


$$
u_{1,0}^{n+\frac{1}{3}}=\frac{\lambda_{2} \widehat{b}_{1,1}-4 \lambda_{2} \widehat{c}_{1,1}}{\widehat{a}_{1,1} \lambda_{2}-3 \widehat{c}_{1,1} \lambda_{2}-2 \Delta x \widehat{c}_{1,1} \beta_{2}} u_{1,1}^{n+\frac{1}{3}}+\frac{-d_{1,1} \lambda_{2}-2 \Delta x \widehat{c}_{1,1} \beta_{2} u_{o c}}{\widehat{a}_{1,1} \lambda_{2}-3 \widehat{c}_{1,1} \lambda_{2}-2 \Delta x \widehat{c}_{1,1} \beta_{2}}
$$

Introducing the notation with (52) we determine:

$$
u_{1,0}^{n+\frac{1}{3}}=\alpha_{1,0} u_{1,1}^{n+\frac{1}{3}}+\beta_{1,0} .
$$

From relation (53), it follows that the sweep coefficients $\alpha_{1,0}$ and $\beta_{1,0}$ are calculated as:

$$
\alpha_{1,0}=\frac{\lambda \widehat{b}_{1,1}-4 \lambda_{2} \widehat{c}_{1,1}}{\widehat{a}_{1,1} \lambda_{2}-3 \widehat{c}_{1,1} \lambda_{2}-2 \Delta x \widehat{c}_{1,1} \beta_{2}} \text { and } \beta_{1,0}=\frac{-d_{1,1} \lambda_{2}-2 \Delta x \widehat{c}_{1,1} \beta_{2} u_{o c}}{\widehat{a}_{1,1} \lambda_{2}-3 \widehat{c}_{1,1} \lambda_{2}-2 \Delta x \hat{c}_{1,1} \beta_{2}}
$$

Now, approximating boundary condition (22) in $x$ and for $i=N$, we obtain:

$$
\lambda_{2} \frac{u_{1, \mathrm{~N}-2}^{n+\frac{1}{3}}-4 u_{1, \mathrm{~N}-1}^{n+\frac{1}{3}}+3 u_{1, \mathrm{~N}}^{n+\frac{1}{3}}}{2 \Delta x}=-\beta_{2} u_{o c}+\beta_{2} u_{1, N}^{n+\frac{1}{3}}
$$

Using the reverse sweep method for the sequence $N, N-1$, and $N-2$, we determine $u_{1, \mathrm{~N}-1}^{n+\frac{1}{3}}$ and $u_{1, \mathrm{~N}-2}^{n+\frac{1}{3}}$

$$
\begin{gathered}
u_{1, \mathrm{~N}-1}^{n+\frac{1}{3}}=\alpha_{1, \mathrm{~N}-1} u_{1, N}^{n+\frac{1}{3}}+\beta_{1, \mathrm{~N}-1} \\
u_{1, \mathrm{~N}-2}^{n+\frac{1}{3}}=\alpha_{1, \mathrm{~N}-2} \alpha_{1, \mathrm{~N}-1} u_{N, j, k}^{n+\frac{1}{3}}+\alpha_{1, \mathrm{~N}-2} \beta_{1, \mathrm{~N}-1}+\beta_{1, \mathrm{~N}-2} .
\end{gathered}
$$

Substituting $u_{1, \mathrm{~N}-1}^{n+\frac{1}{3}}$ from (55) and $u_{1, \mathrm{~N}-2}^{n+\frac{1}{3}}$ from (56) into (54) we determine $u_{1, \mathrm{~N}}^{n+\frac{1}{3}}$ :

$$
u_{1, \mathrm{~N}}^{n+\frac{1}{3}}=\frac{-\lambda_{2} \alpha_{1, \mathrm{~N}-2} \beta_{1, \mathrm{~N}-1}-\lambda_{2} \beta_{1, \mathrm{~N}-2}+4 \lambda_{2} \beta_{1, \mathrm{~N}-1}-2 \Delta x \beta_{2} u_{o c}}{3 \lambda_{2}-2 \Delta x \beta_{2}+\lambda_{2} \alpha_{1, \mathrm{~N}-2} \alpha_{1, \mathrm{~N}-1}-4 \lambda_{2} \alpha_{1, \mathrm{~N}-1}} .
$$

The values of the moisture sequence $u_{1, \mathrm{~N}-1}^{n+\frac{1}{3}}, u_{1, \mathrm{~N}-2}^{n+\frac{1}{3}}, \ldots, u_{1,1}^{n+\frac{1}{3}}$ are determined by the reverse sweep method by decreasing $i$ sequence:

$$
u_{1, i}^{n+\frac{1}{3}}=\alpha_{1, i} u_{1, i+1}^{n+\frac{1}{3}}+\beta_{1, i}, \text { where } i=\overline{N-1.1} .
$$


Similarly, using the above method, for problem B, we approximate in $y$, and for problem $\mathrm{C}$ we approximate in $z$ and obtain the values of the temperature and moisture sequence by the reverse sweep method.

\section{Results and Discussion}

\subsection{Computational experiment}

Based on the developed numerical algorithm, a software tool in the C\# language was compiled to conduct a computational experiment (CE) on a computer (Figs. 1).

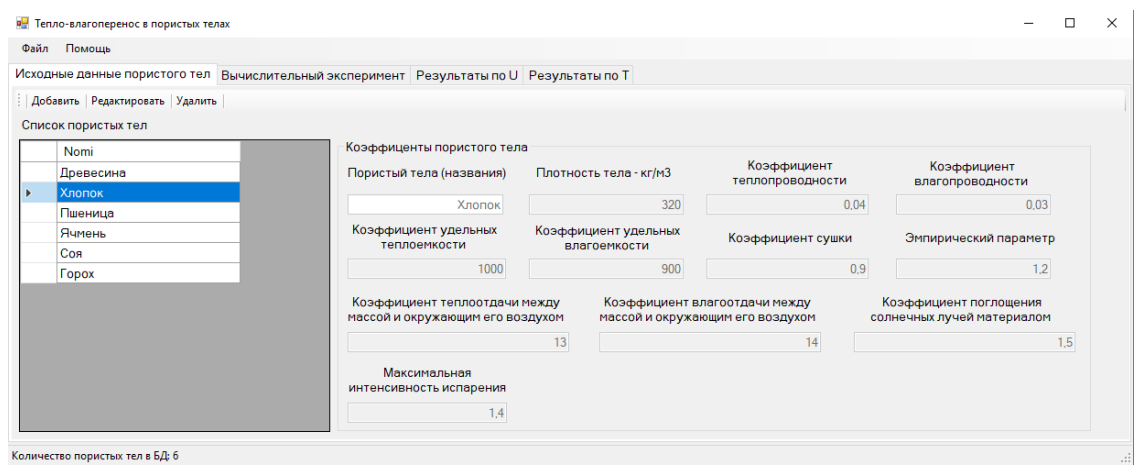

Fig.1. Program interface for entering basic parameters of the object under research

Numerical calculations were conducted for the following data for raw cotton seeds: the thermal conductivity coefficient is $0.04 \mathrm{Vt}^{-1} \mathrm{~K}^{-1}$, the density is $320 \mathrm{~kg} \mathrm{~m}^{-3}$, the specific heat capacity is $1000 \mathrm{~J} \mathrm{~kg}^{-1} \mathrm{~K}^{-1}$, the corresponding thermal diffusivity is $a^{2}=1.25 \cdot 10^{-7} \mathrm{~m}^{2} \mathrm{~s}^{-1}$. In the main calculations, the heat release coefficient is $b=1.0 \cdot 10^{-5} \mathrm{~K} \mathrm{~s}^{-1}$, the coefficient in the heat release exponent is $\alpha=4.0 \cdot 10^{-5} s^{-1}$. The heat transfer coefficient ratio to the thermal conductivity coefficient is taken as $k=1 s^{-1}$. The sizes of the raw cotton pile are taken as $L_{x}=15 \mathrm{~m} ; L_{y}=12 \mathrm{~m} ; L_{z}=8 \mathrm{~m} ;$ the initial temperature and moisture of the porous body are taken as $T_{0}=30^{\circ} \mathrm{C}$ and $u_{0}=60 \%$, respectively. Computational experiments were carried out in September 2020 in the city of Bukhara, in which ambient the average temperature was $T_{o c}=25^{\circ} \mathrm{C}$ (during the day $+26^{\circ} \mathrm{C}$, at night $+23^{\circ} \mathrm{C}$ ) and the average ambient humidity was $u_{o c}=36 \%$.

The results of the numerical calculations performed on a computer are shown in Figs. 2 -11 . 


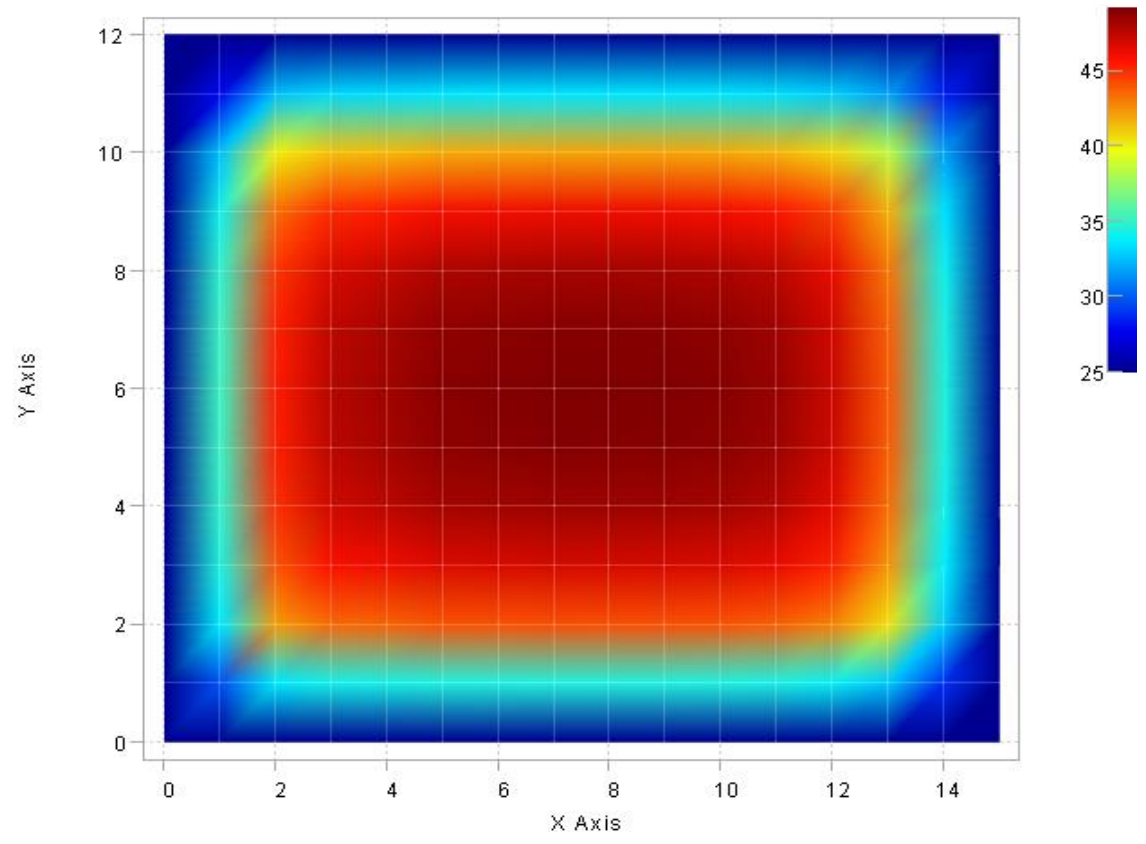

Fig. 2. Changes in temperature in a raw cotton pile at $\tau=10$ days (pile temperature in degrees).

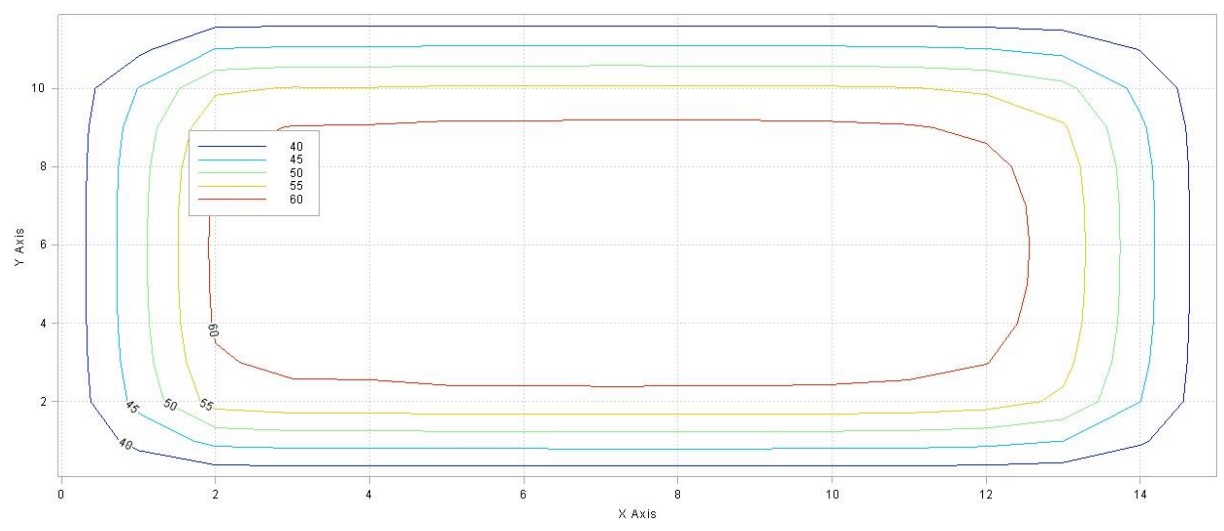

Fig. 3. Change in moisture content in a raw cotton pile at $\tau=10$ days (moisture content in percent)

Figures 2 - 3 show the results of CE to determine the change in temperature and moisture content in the section $\mathrm{z}=4 \mathrm{~m}$ over time. Based on the results of $\mathrm{CE}$, we can say that inside the raw cotton pile, the temperature increases exponentially with time and is $48^{\circ} \mathrm{C}$ in the inner layer of the cotton pile. The moisture content inside the pile insufficiently increases as compared to the outer layer of the cotton pile and reaches its maximum value in the center of the section $\mathrm{z}=4 \mathrm{~m}$ and is $60 \%$. 


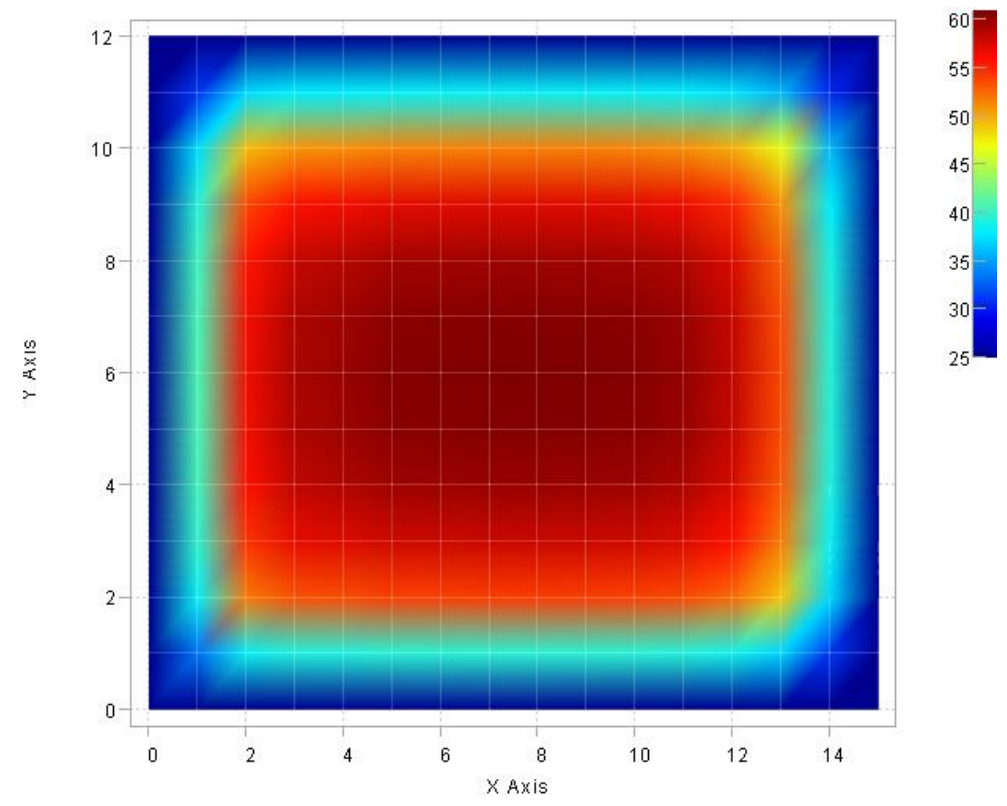

Fig. 4. Change in temperature in a raw cotton pile at $\tau=15$ days (pile temperature in degrees)

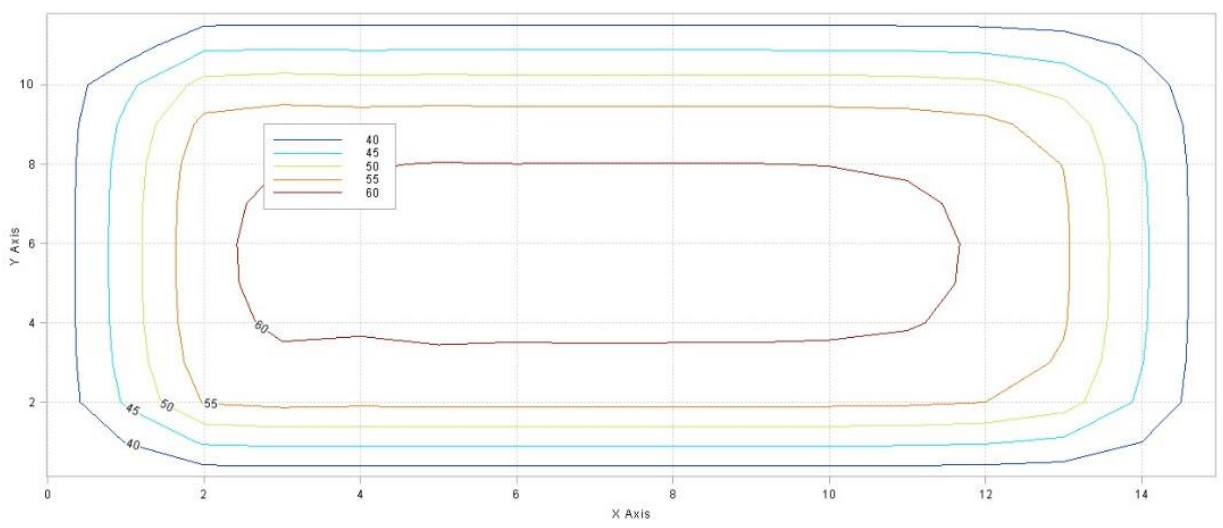

Fig. 5. Change in moisture content in a raw cotton pile at $\tau=15$ days (moisture content in percent)

Figures 4-5 show the CE results to determine the change in temperature and moisture content over time in a cotton pile in the section $\mathrm{z}=4 \mathrm{~m}$. From the analysis of the numerical calculations performed, it follows that when the pile is stored for 15 days, the temperature of its mass reaches $61^{\circ} \mathrm{C}$, and the moisture content inside the pile increases to $55-60 \%$. 


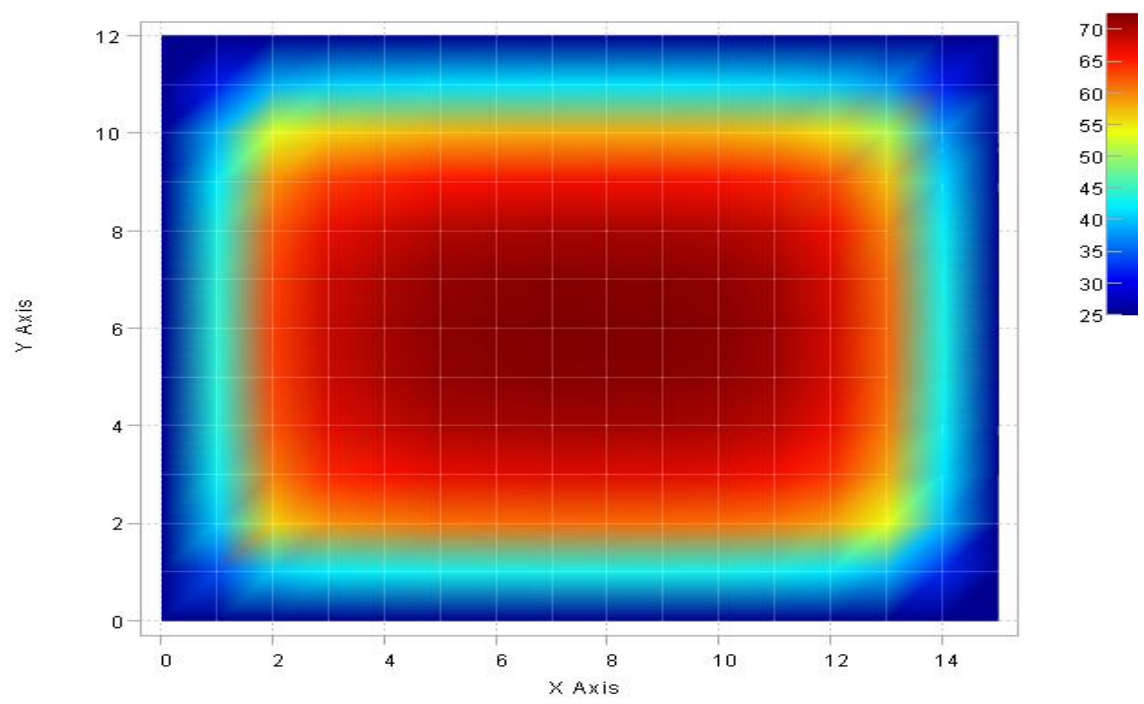

Fig. 6. Changes in temperature in a raw cotton pile at $\tau=20$ days (pile temperature in degrees)

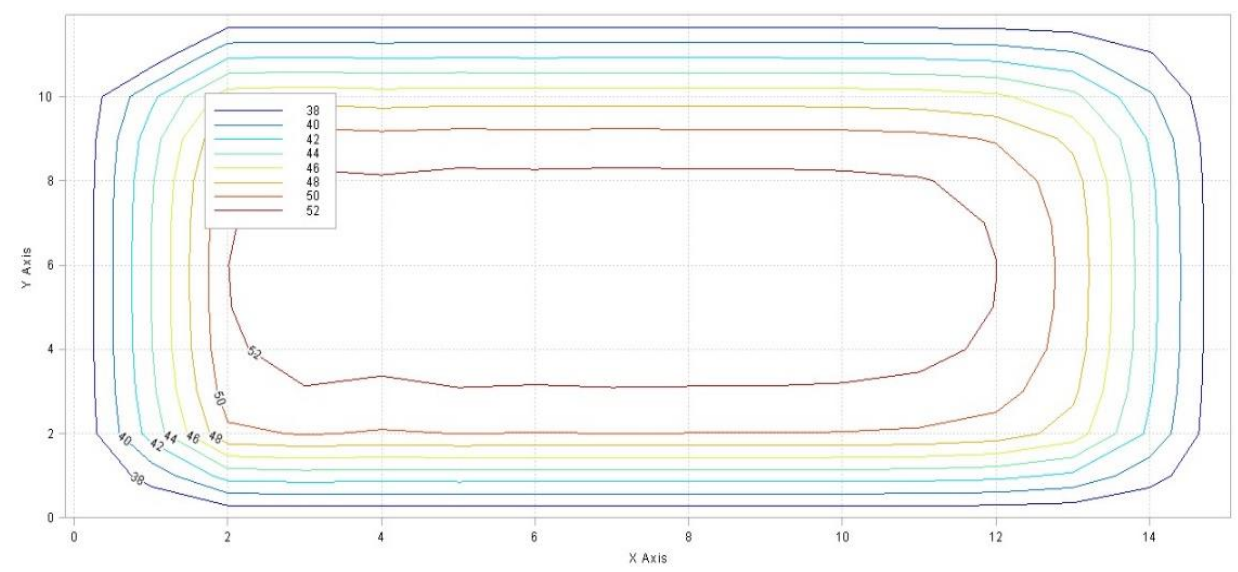

Fig.7. Change in moisture content in a raw cotton pile at $\tau=20$ days (moisture content in percent)

Figures $6-7$ show the results of $\mathrm{CE}$ to determine the change in temperature and moisture content of a cotton pile in the section $z=4 \mathrm{~m}$ over time. From the analysis of the calculations obtained, it follows that after 20 days of storing a cotton pile, the temperature of the pile increases linearly over time, and the moisture content remains almost unchanged and is $52 \%$. 


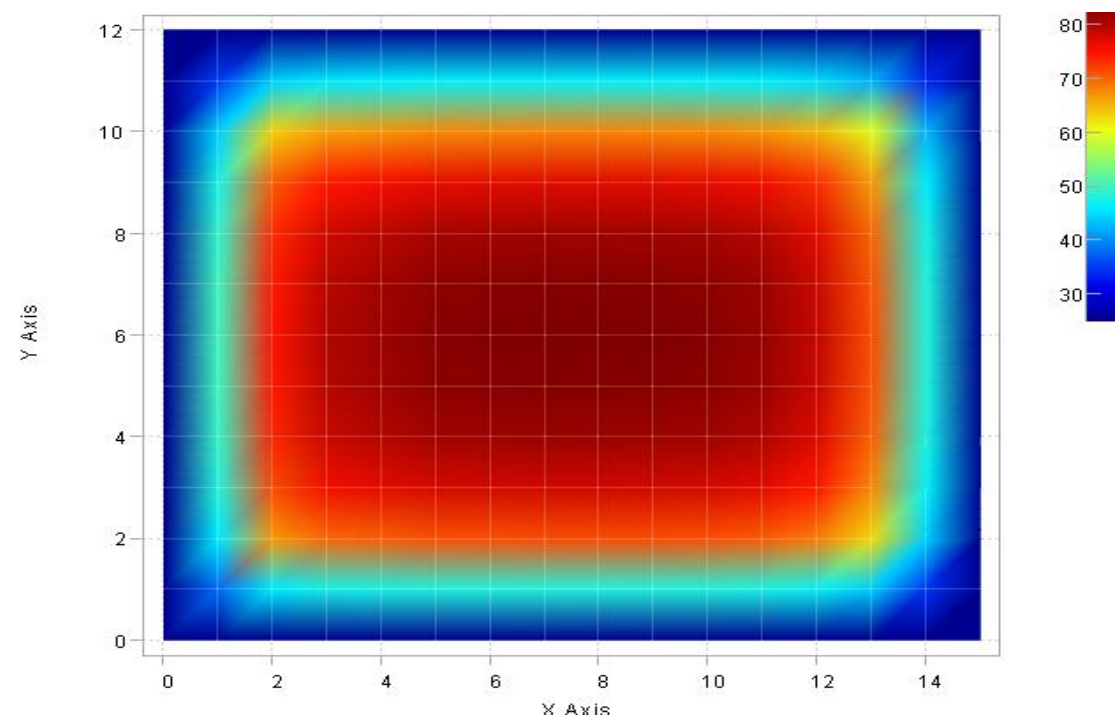

Fig. 8. Change in temperature in a raw cotton pile at $\tau=25$ days.

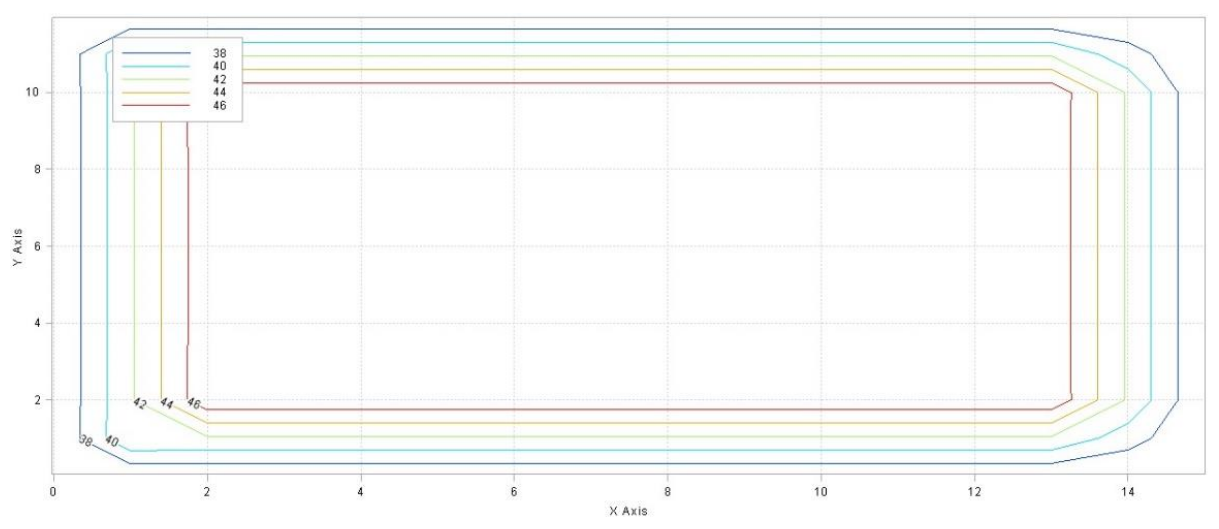

Fig. 9. Change in moisture content in a raw cotton pile at $\tau=25$ days.

Figures 8 -9 show the numerical calculations on a computer with given initial values of temperature and moisture content of the pile. As seen from the analysis of numerical calculations, the main increase in temperature and moisture content is observed in the inner layers of the cotton pile. Numerical calculations were also conducted with various values of ambient temperature and humidity. In $\mathrm{CE}$, it was established that heat and moisture exchange with the environment occurs in the upper layers of the cotton pile, and in the inner layer, an increase in temperature and moisture content occurs due to cotton respiration; it depends on the moisture content of the piled raw materials. 


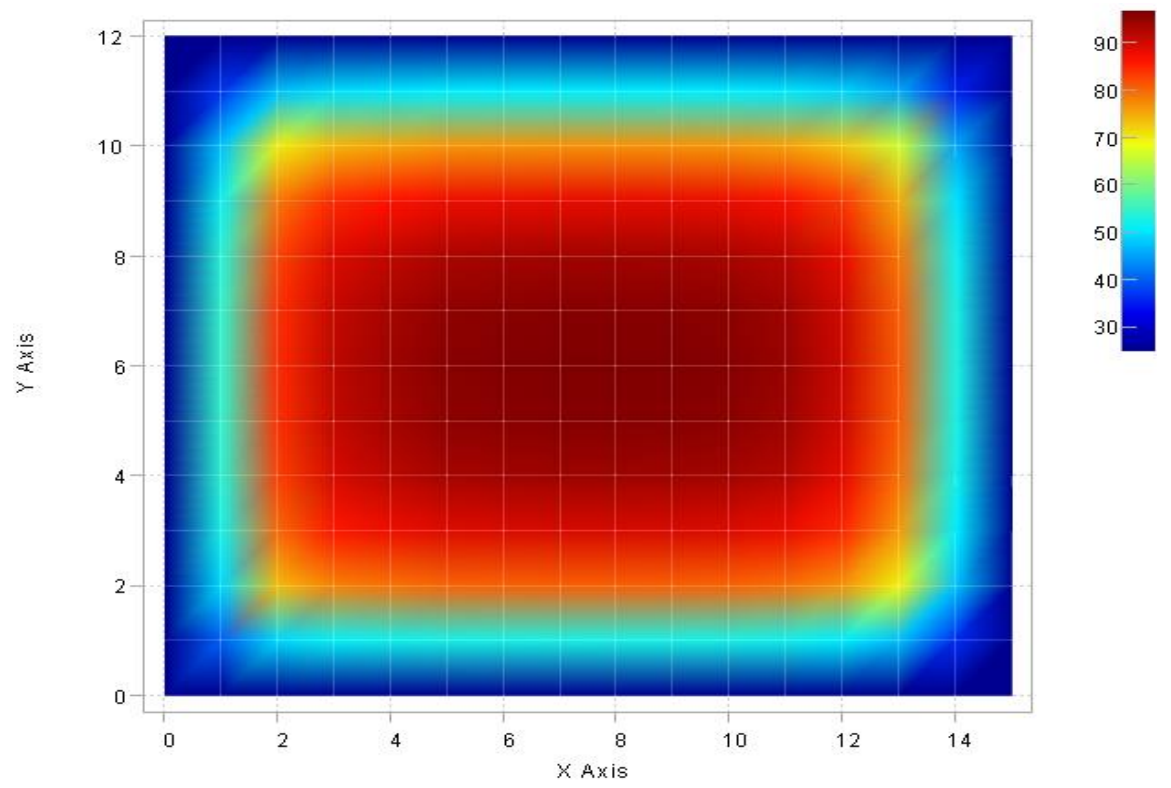

Fig. 10. Change in temperature in a raw cotton pile at $\tau=30$ days.

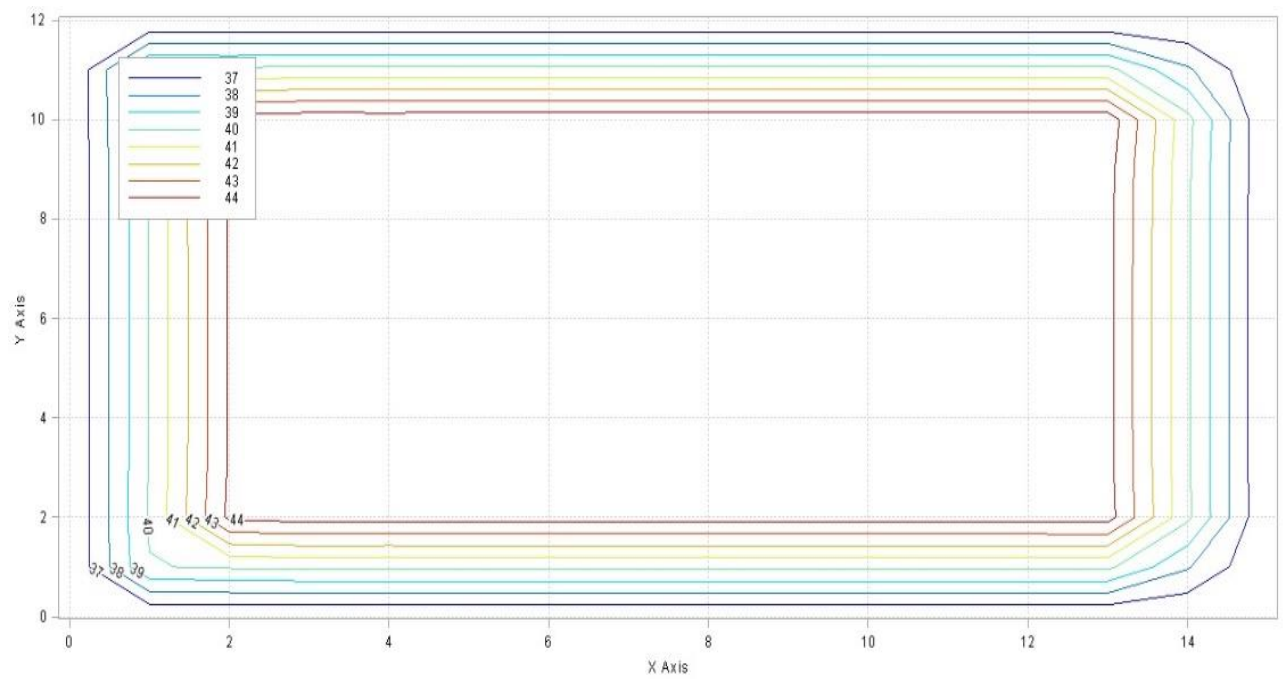

Fig. 11. Change in moisture content in a raw cotton pile at $\tau=30$ days

As shown in Figs. 10 - 11 during long-term storage (30 days), the temperature rises to $95-96^{\circ} \mathrm{C}$, and the moisture content decreases to $44 \%$ in the inner layers of the pile. With an increase in the moisture content of raw cotton, the values of thermal diffusivity and the intensity of heat release increase. This leads to more intense heating of the inner part of the pile.

Analyzing the results of numerical calculations, we can conclude that the factor of internal moisture and heat release becomes substantial and can lead to spontaneous combustion of the pile or to the loss of natural qualities of raw cotton during long-term storage. 


\section{Conclusions}

A numerical experiment and analysis of its results are conducted based on the developed three-dimensional mathematical model of the process of heat and moisture transfer during storage and drying of porous bodies. The software was developed for calculating the process of heat and moisture transfer in natural products under internal heat release and external temperature, caused by the influence of solar energy, which makes it possible to determine changes in the thermophysical parameters of the object under study.

The numerical calculations performed have established that moisture and heat transfer and their exchange with the environment occurs in the upper layers of the cotton pile, and in the inner layer, temperature and moisture increase due to the respiration of cotton and seeds, which depends on the moisture content of the raw cotton pile and the products of hulling.

From the analysis of the performed numerical calculations, it was established that with an increase in the moisture content of raw cotton, the values of thermal diffusivity and the intensity of heat release increase proportionally, leading to more intense heating of the inner part of the pile.

Analysis of the results of numerical calculations showed that during long-term storage (30 days and more, without ventilation), the temperature inside the cotton pile heats more than $85^{\circ} \mathrm{C}$, and the moisture content inside the pile changes to $44 \%$, which can lead to spontaneous combustion of the pile or to the loss of natural qualities of raw cotton exposed to solar radiation.

\section{References}

1. J. R. Philip and D. A. De Vries, Moisture movement in porous materials under temperature gradients, Trans. Am. Geophys. Union, 38, (2), p. 222, 1957, doi: 10.1029/TR038i002p00222.

2. A. V. Lykov, On systems of differential equations for heat and mass transfer in capillary porous bodies, J. Eng. Phys., 26, (1), pp. 11-17, Jan. 1974, doi: 10.1007/BF00827280.

3. S. Whitaker, Simultaneous Heat, Mass, and Momentum Transfer in Porous Media. A Theory of Drying', pp. 119-203. (1977).

4. A. V. Lykov and Y. A. Mikhailov, Theory of Heat and Mass Transfer. Gosenergoizdat, 1963.

5. A. V. Lykov, Theory of drying [in Russian]. Moscow: Énergiya, (1973).

6. Y. Irudayaraj, Joseph; Wu, Analysis and application of Luikov's heat, mass, and pressure transfer model to a capillary porous media, Dry. Technol., 14, (3-4), pp. 803-824, (1996).

7. R. W. Lewis and W. J. Ferguson, The effect of temperature and total gas pressure on the moisture content in a capillary porous body, Int. J. Numer. Methods Eng., 29, (2), pp. 357-369, Feb. (1990), doi: 10.1002/nme.1620290210.

8. A. Afanasyev and B. Siplivyy, Algorithm for the Joint Solution of Heat and Mass Transfer Equations and Equations of the Electromagnetic Field During the Drying of Microwave Radiation, Vestn. Volgogr. Gos. Univ. Ser. 1. Math. Phys., 2, (2), pp. 8293, Mar. (2017), doi: 10.15688/jvolsu1.2017.2.8.

9. R. N. Pandey, S. K. Srivastava, and M. D. Mikhailov, Solutions of Luikov equations of heat and mass transfer in capillary porous bodies through matrix calculus: a new approach, Int. J. Heat Mass Transf., 42, (14), pp. 2649-2660, Jul. (1999), doi: 10.1016/S0017-9310(98)00253-1.

10. R. Younsi, D. Kocaefe, and Y. Kocaefe, Three-dimensional simulation of heat and 
moisture transfer in wood, Appl. Therm. Eng., 26, (11-120, pp. 1274-1285, Aug. (2006), doi: 10.1016/j.applthermaleng.2005.10.029.

11. G. Akbari, H. Basirat Tabrizi, and E. Damangir, Numerical and experimental investigation of variable phase transformation number effect in porous media during freezing process, Heat Mass Transf., 45, (4), pp. 407-416, Feb. (2009), doi: 10.1007/s00231-008-0444-y.

12. M. Asli, F. Brachelet, A. Chauchois, E. Antczak, and D. Defer, Numerical and experimental investigation of heat and mass transfer within bio-based material, Therm. Sci., 23, (1), pp. 23-31, (2019), doi: 10.2298/TSCI161019175A.

13. Z. Q. Wang Huilin, Lu Tao, Drying characteristics of biological porous media during convective drying, Int J Agric Biol Eng, 9, (5), pp. 194-207, (2016), doi: 10.3965/j.ijabe.20160905.2057.

14. W. Cai, L. Zhu, S. Dong, G. Xie, and J. Li, Effect of Thermophysical Properties on Coupled Heat and Mass Transfer in Porous Material during Forced Convective Drying, Adv. Mech. Eng., 6, p. 830387, Jan. (2014), doi: 10.1155/2014/830387.

15. S. M. A. Rahman, M. R. Islam, and A. S. Mujumdar, A Study of Coupled Heat and Mass Transfer in Composite Food Products during Convective Drying, Dry. Technol., 25, (7-8), pp. 1359-1368, Aug. (2007), doi: 10.1080/07373930701438956.

16. A. Modak, H. Zuo, S. S. Takagaki, and S. Ramaswamy, 'The Role of Nonuniformity in Convective Heat and Mass Transfer through Porous Media, Part 1', Dry. Technol., 29, ( 5), pp. 536-542, Mar. (2011), doi: 10.1080/07373937.2010.513781.

17. V. D. Thi, M. Li, M. Khelifa, M. El Ganaoui, and Y. Rogaume, Finite Element Modeling of Heat and Moisture Transfer in Porous Material, World Acad. Sci. Eng. Technol. Int. J. Mater. Metall. Eng., 11, (4), pp. 345-351, (2017), doi: 10.5281/zenodo.1130363.

18. S. S. Makarov, Modeling heat and mass transfer in a bubble in a liquid during spray cooling of a high-temperature metal, Bull. Udmurt Univ. Maths. Mech. Comput. Sci., 4, pp. 96-100, (2010).

19. N. Ravshanov, I. U. Shadmanov, and O. J. Kravets, Mathematical model for the study and prediction of a porous body thermal state, IOP Conf. Ser. Mater. Sci. Eng., 537, (2), (2019), doi: 10.1088/1757-899X/537/2/022024.

20. N. Ravshanov and I. U. Shadmanov, Multidimensional model of heat-moisture transport in porous media, J. Phys. Conf. Ser., 1546, p. 012098, May (2020), doi: 10.1088/1742-6596/1546/1/012098.

21. N. N. Yanenko, The Method of Fractional Steps, Nauka. Berlin, Heidelberg: Springer Berlin Heidelberg, (1971).

22. A. A. Samarskii, P. P. Matus, and P. N. Vabishchevich, Difference Schemes with Operator Factors. Dordrecht: Springer Netherlands, (2002).

23. G. I. Marchuk, Splitting methods, Nauka. Moscow, (1988).

24. A. Krainov and L. Minkov, Numerical methods for solving problems of heat and mass transfer, Teaching aid. (2016).

25. A. Voevodin and O. Goncharova, Method for calculating two-dimensional convection problems based on splitting into physical processes, Comput. Technol., 1, (7), pp. 6975,(2002).

26. O. Goncharova, Method of splitting into physical processes for calculating threedimensional convection problems, Bull. Altai State Univ., 1, pp. 39-44, (2007). 